\title{
$3-527-2$
}

\section{Molecular pathogenesis of autism spectrum disorder with abnormal chromatin remodeling}

\section{Yuta Katayama}

Dept. of Mol. and Biol., Med. Ins. of Bioreg., Univ. of Kyushu

Autism spectrum disorder (ASD) comprises a range of neurodevelopmental disorders characterized by deficits in social interaction and communication as well as by restricted and repetitive behaviours. ASD has a strong genetic component with high heritability. Exome sequencing analysis has identified many de novo mutations in a variety of genes in individuals with ASD, with CHD8 being most frequently affected. CHD8 is a member of the chromodomain helicase DNA-binding (CHD) family of proteins and functions as an ATP-dependent chromatin-remodeling factor in regulation of the expression of many genes including those for b-catenin and p53.

We showed that mice heterozygous for Chd8 mutations manifest ASD-like behavioural characteristics including increased anxiety, persistence tendency, and altered social behaviour. Unexpectedly, transcriptome analyses revealed that CHD8 haploinsufficiency did not result in prominent changes in the expression of a few specific genes but rather gave rise to small but global changes in gene expression with neurodevelopmental delay in the mutant mouse embryos. Furthermore, reduced expression of CHD8 is associated with abnormal activation of RE-1 silencing transcription factor (REST), which suppresses the transcription of many neuronal genes. REST activation was also observed in the human ASD brain. Our results are thus consistent with the notion that CHD8 haploinsufficiency is a highly penetrant risk factor for ASD, with disease pathogenesis likely resulting from a delay in neurodevelopment.

Our recent research has focused on the molecular mechanism and identification of responsible cell types, and would like to introduce the recently elucidated mechanism of ASD with CHD8 mutation. 